\title{
Nonreciprocal spin pumping damping in asymmetric magnetic trilayers
}

\author{
Yevgen Pogoryelov, ${ }^{1}$ Manuel Pereiro, ${ }^{1}$ Somnath Jana,,${ }^{1}$ Ankit Kumar, ${ }^{2}$ Serkan Akansel, ${ }^{2}$ Mojtaba Ranjbar,,${ }^{3}$ Danny Thonig, ${ }^{4}$ \\ Daniel Primetzhofer, ${ }^{1}$ Peter Svedlindh, ${ }^{2}$ Johan Åkerman, ${ }^{3,5}$ Olle Eriksson, ${ }^{1,4}$ Olof Karis, ${ }^{1}$ and Darío A. Arena ${ }^{6, *}$ \\ ${ }^{1}$ Department of Physics and Astronomy, Uppsala University, 75121 Uppsala, Sweden \\ ${ }^{2}$ Department of Engineering Sciences, Uppsala University, 75121 Uppsala, Sweden \\ ${ }^{3}$ Department of Physics, University of Gothenburg, 41296 Gothenburg, Sweden \\ ${ }^{4}$ School of Science and Technology, Örebro University, 70182 Örebro, Sweden \\ ${ }^{5}$ Materials and Nanophysics, School of ICT, KTH Royal Institute of Technology, 16400 Kista, Sweden \\ ${ }^{6}$ Department of Physics, University of South Florida, Tampa, Florida 33620, USA
}

(Received 16 July 2019; revised manuscript received 14 January 2020; accepted 14 January 2020; published 3 February 2020)

\begin{abstract}
In magnetic trilayer systems, spin pumping is generally addressed as a reciprocal mechanism characterized by one unique spin-mixing conductance common to both interfaces. However, this assumption is questionable in cases where different types of interfaces are present. Here, we present a general theory for analyzing spin pumping in cases with more than one unique interface and where the magnetic coupling is allowed to be noncollinear. The theory is applied to analyze layer-resolved ferromagnetic resonance experiments on the trilayer system $\mathrm{Ni}_{80} \mathrm{Fe}_{20} / \mathrm{Ru} / \mathrm{Fe}_{49} \mathrm{Co}_{49} \mathrm{~V}_{2}$ where the $\mathrm{Ru}$ spacer thickness is varied to tune the indirect exchange coupling. It is demonstrated that the equation of motion of macrospins driven by spin pumping need to be modified in case of noncollinear coupling. Our analysis also shows that the spin pumping in trilayer systems with dissimilar magnetic layers, in general, is nonreciprocal.
\end{abstract}

DOI: 10.1103/PhysRevB.101.054401

\section{INTRODUCTION}

Spin transport in thin-film heterostructures can generate a rich spectrum of physical effects [1-9] and has great potential for realizing new spintronic functionality $[10,11]$. Pure spin currents, which can modify the dynamics of magnetic multilayers, can be generated in metallic ferromagnetic (FM)/nonmagnetic (NM) heterostructures via the spin pumping process [12]. Precessing spins in the FM generate a spin current that can diffuse away from the FM/NM interface and into the NM layer. Propagation of spin currents in the heterostructure can lead to spin accumulation in the NM material [13], spin to charge conversion in a NM layer [14], increased Gilbert-like damping [15], and nonlocal perturbation of a second FM layer [16].

The efficiency of the spin-pumping process across a FM/NM interface is typically parametrized by the spinmixing conductance $g^{\uparrow \downarrow}$ which relates the additional damping from spin pumping to the film thickness and intrinsic properties $[12,17]$. In magnetic trilayer structures (FM1/NM/FM2), spin pumping is often treated as a reciprocal process (FM1 $\stackrel{\text { SP }}{\Leftrightarrow}$ FM2), characterized by a single $g^{\uparrow \downarrow}$ common to both interfaces; this approach works well when FM1 and FM2 are the same material that generates an equivalent FM/NM interface $[18,19]$. Many spintronic devices rely on layered magnetic structures where FM1 and FM2 are different materials [20-25] with different interfaces on each side of the spacer layer. In several cases, these systems have more complex interlayer exchange, such as biquadratic exchange, combined

*Corresponding author: darena@usf.edu with noncollinear magnetic configurations. This calls for an analytical framework to analyze magnetization dynamics of such systems where such complexities are explicitly considered. We provide such a theoretical model in this article together with experimental data.

The model proposed accounts for FM1 and FM2 layers with different intrinsic parameters. Two key features of the treatment are as follows: (a) the separation of the spin-mixing conductance into distinct contributions for the two dissimilar interfaces, and (b) a self-consistent macrospin framework for noncolinear magnetizations. We apply this theory to analyze layer-resolved ferromagnetic resonance (FMR) experiments measured with $\mathrm{x}$-ray-detected FMR (X-FMR) from magnetic trilayer samples where the NM spacer thickness is varied to tune the indirect exchange coupling. The analysis demonstrates that the spin-pumping damping from FM1 into FM2 is nonreciprocal with the spin-pumping damping in the reverse direction. The nonreciprocity is significant, which may enable new spintronics technologies.

\section{NONRECIPROCAL SPIN PUMPING DAMPING FRAMEWORK}

We consider a trilayer system involving permalloy $\left(\mathrm{Py}-\mathrm{Ni}_{80} \mathrm{Fe}_{20}\right), \mathrm{Ru}$, and permendur (Pmd- $\mathrm{Fe}_{49} \mathrm{Co}_{49} \mathrm{~V}_{2}$ ). In the $\mathrm{Py} / \mathrm{Ru} / \mathrm{Pmd}$ system, the Py and Pmd layers provide large magnetic moments, whereas $\mathrm{Ru}$ is the NM spacer. In the following, the first magnetic layer (Py) is labeled by 1 , and the second magnetic layer is labeled by 2 (Pmd) as shown in Fig. 1. We assume that each layer can be represented by a single $\boldsymbol{m}_{i}$ and $\boldsymbol{m}_{j}$ (macrospin approximation), where $i, j=1,2$. 


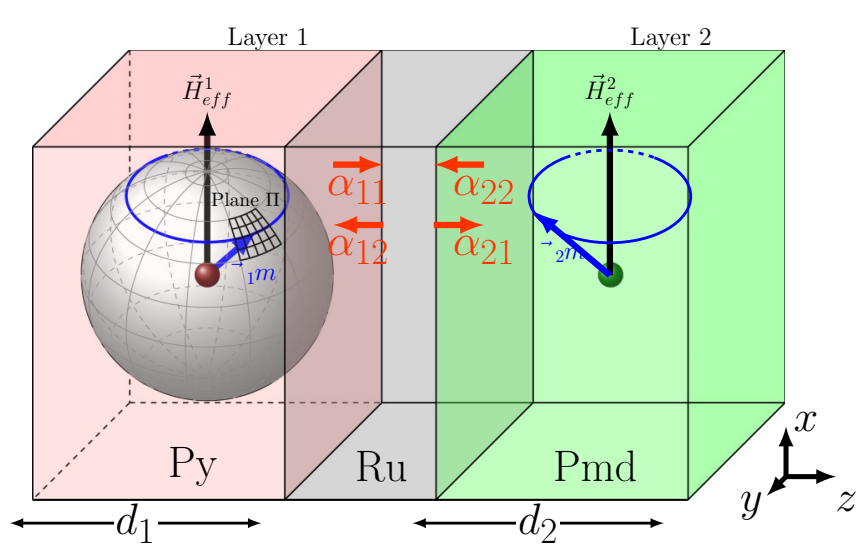

FIG. 1. Trilayer system composed of permalloy (1) and permendur (2) layers separated by a ruthenium spacer. The coordinate system is shown on the bottom right. The blue arrow represents the precession of the spin on the $\widehat{y}-\widehat{z}$ plane, and the black arrow indicates the direction of the external magnetic field. The sphere represents the locus of constant magnetization length whereas $\Pi$ stands for the plane tangential to the surface of the sphere. The red-yellow arrows indicate the local $\left(\alpha_{i i}\right)$ and interlayer $\left(\alpha_{i j}\right)$ spin-pumping damping due to the currents flowing in and out of the layers. The magnetic layer's thickness is represented by $d_{1}$ and $d_{2}$.

The equation of motion of the macrospins can be recast as

$$
\begin{aligned}
\frac{\partial \boldsymbol{m}_{i}}{\partial t}= & -\gamma \boldsymbol{m}_{i} \times \boldsymbol{H}_{\mathrm{eff}}^{i}+\left(\alpha_{i}^{0}+\alpha_{i i}^{s p}\right) \boldsymbol{m}_{i} \times \frac{\partial \boldsymbol{m}_{i}}{\partial t} \\
& -\frac{\alpha_{i j}^{s p}}{\left|\boldsymbol{m}_{i}\right|^{2}} \boldsymbol{m}_{i} \times\left(\boldsymbol{m}_{j} \times \frac{\partial \boldsymbol{m}_{j}}{\partial t}\right) \times \boldsymbol{m}_{i}
\end{aligned}
$$

where $\gamma \approx 3.5 \times 10^{-5} \mathrm{GHz}^{-1} \mathrm{~m}^{-1} \mathrm{~A}$ is the gyromagnetic ratio. Both macrospins $\left(\boldsymbol{m}_{i}, i=1,2\right)$ precess around their effective fields $\boldsymbol{H}_{\text {eff }}^{i}=-\partial \mathcal{H} / \partial \boldsymbol{m}_{i}$, where $\mathcal{H}$ is the spin Hamiltonian. The nonlocal interlayer damping $\alpha_{i j}^{s p}$ accounts for spin-pumping contributions into layer $i$ from layer $j$, whereas the local damping $\alpha_{i i}^{s p}$ parametrizes spin pumping out of layer $i$. The influence of the spin-pumping damping $\left(\alpha_{i j}^{s p}\right)$ on the model is discussed in Appendix A. The intrinsic damping of layer $i$ is given by $\alpha_{i}^{0}$. The last term is of a form previously not discussed and represents the influence of nonlocal dynamic spin pumping of a noncollinear magnetic arrangement between two ferromagnetic layers of a trilayer system. This term describes a projection of the vector $\boldsymbol{m}_{j} \times \frac{\partial \boldsymbol{m}_{j}}{\partial t}$ from layer $j$ over plane $\Pi$ tangential to the sphere circumscribed by the macrospin $i$ as shown in Fig. 1. The last term of Eq. (1) fulfills the requirement that the rate of change of magnetization $\left(\frac{\partial \boldsymbol{m}_{i}}{\partial t}\right)$ is perpendicular to the magnetization $\left(\boldsymbol{m}_{i}\right)$ at all times $\left(\boldsymbol{m}_{i} \cdot \frac{\partial \boldsymbol{m}_{i}}{\partial t}\right)=0$.

The coupled trilayer system in the coordinate system indicated in Fig. 1 is described by the following Hamiltonian $\mathcal{H}$ :

$$
\begin{aligned}
\mathcal{H}= & -\frac{A_{e x}}{\mu_{0} d_{i}} \frac{\boldsymbol{m}_{i} \cdot \boldsymbol{m}_{j}}{\left|\boldsymbol{m}_{i}\right|\left|\boldsymbol{m}_{j}\right|}-\frac{B_{e x}}{\mu_{0} d_{i}} \frac{\left(\boldsymbol{m}_{i} \cdot \boldsymbol{m}_{j}\right)^{2}}{\left|\boldsymbol{m}_{i}\right|^{2}\left|\boldsymbol{m}_{j}\right|^{2}}+\frac{1}{2} \overline{\bar{N}}_{i} \boldsymbol{m}_{i}^{2} \\
& -H_{0} \widehat{\boldsymbol{x}} \cdot \boldsymbol{m}_{i}+\frac{1}{2} \delta_{i} \widehat{\boldsymbol{x}} \cdot \boldsymbol{m}_{i}-h \widehat{\boldsymbol{y}} \cdot \boldsymbol{m}_{i}
\end{aligned}
$$

$$
\begin{aligned}
& +\frac{K_{1 i}^{u}}{\mu_{0}\left|\boldsymbol{m}_{i}\right|^{2}}\left(\boldsymbol{m}_{i} \cdot \widehat{\boldsymbol{e}}_{u}\right)^{2}+\frac{K_{1 i}^{c}}{\mu_{0}\left|\boldsymbol{m}_{i}\right|^{4}}\left[\left(\boldsymbol{m}_{i} \cdot \widehat{\boldsymbol{x}}\right)^{2}\left(\boldsymbol{m}_{i} \cdot \widehat{\boldsymbol{y}}\right)^{2}\right. \\
& \left.+\left(\boldsymbol{m}_{i} \cdot \widehat{\boldsymbol{y}}\right)^{2}\left(\boldsymbol{m}_{i} \cdot \widehat{\boldsymbol{z}}\right)^{2}+\left(\boldsymbol{m}_{i} \cdot \widehat{\boldsymbol{z}}\right)^{2}\left(\boldsymbol{m}_{i} \cdot \widehat{\boldsymbol{x}}\right)^{2}\right],
\end{aligned}
$$

where the first $\left(A_{e x}\right)$ and second $\left(B_{e x}\right)$ terms represent the bilinear and biquadratic exchange energies, respectively. The parameter $d_{i}$ indicates the thickness of the ferromagnetic layer whereas $\mu_{0} \approx 4 \pi \times 10^{-7} \mathrm{~J} \mathrm{~m}^{-1} \mathrm{~A}^{-2}$ is the vacuum permeability. The next term is the demagnetization energy. In a thin film with $x, y \gg z$ and with the magnetization in-plane ( $x$-y plane), the demagnetization tensor $\overline{\bar{N}}_{i}$ is almost zero and will not be considered hereafter. The fourth term $\left(H_{0}\right)$ represents the energy of a static external magnetic field along the $\widehat{x}$ direction whereas the following term is the magnetic dipolar field where $\delta_{i}$ is a term that depends on the structural parameters of the layer as shown in Ref. [26]. We consider here only the influence of the field along the $\widehat{x}$ direction since the dipolar field at a distant point $(0,0, z)$ has only the $x$ component for the field. The next term is the microwave field $h=\tilde{h} e^{-i \omega t}$ oscillating at a frequency $\omega$ along the $\widehat{y}$ direction in the experimental setup. Finally, the last two terms represent the uniaxial (with the easy axis $\boldsymbol{e}_{u}$ ) and cubic magnetocrystalline anisotropy energy, respectively.

Since the moments rotate around the external magnetic field, the condition $m^{x} \gg m^{y}, m^{z}$ is fulfilled; hence, $\frac{d m_{i}^{x}}{d t}=0$. Moreover, the sinusodial microwave field induces a magnetization that conveniently can be expressed in a complex form. Thus, $m_{i}^{y}(t)=\tilde{m}_{i}^{y} \exp \left[i\left(\phi_{i}+\omega_{i} t\right)\right], m_{i}^{z}(t)=$ $\tilde{m}_{i}^{z} \exp \left[i\left(\phi_{i}^{\prime}+\omega_{i} t\right)\right]$ with $\phi_{i}^{\prime}=\phi_{i}+\pi / 2$ and $\omega=\omega_{i}$. The phase $\phi_{i}$ at time $t=0$ of layer $i$ is a function of the external magnetic field and is measured by X-FMR experiments [see Figs. 2(d)-2(f)]. Assuming that the angle of precession of the macrospin is relatively small $\left(m^{x} \gg m^{y}, m^{z}\right)$ and that $h \ll H_{0}$, it is a good approximation to linearize the equations of motion. We retain only the terms linear in $h, m^{y}$, and $m^{z}$. By inserting Eq. (2) into Eq. (1) through the definition of the effective field, the linearized coupled equations of motion for both magnetic layers are given by

$$
\overline{\bar{\chi}}\left(\begin{array}{c}
0 \\
\tilde{h} \\
0 \\
\tilde{h}
\end{array}\right)=\left(\begin{array}{c}
\tilde{m}_{1}^{y} \\
\tilde{m}_{1}^{z} \\
\tilde{m}_{2}^{y} \\
\tilde{m}_{2}^{z}
\end{array}\right),
$$

where the elements of the magnetic susceptibility matrix $\overline{\bar{\chi}}$ are shown in Appendix B. The dimensionless intrinsic Gilbert damping parameter is defined as $\eta_{i}^{0}=m_{i}^{x} \alpha_{i}^{0}$. The spinpumping damping parameter out of layer $i$ is $\eta_{i i}^{s p}=m_{i}^{x} \alpha_{i i}^{s p}$, whereas the dimensionless spin backflow into layer $i$ from layer $j$ is defined as $\eta_{i j}^{s p}=m_{j}^{x} \alpha_{i j}^{s p}$. The amplitude of the macrospin precession shown in Figs. 2(a)-2(c) is calculated from the four-index susceptibility matrix [27] of the system $\overline{\bar{\chi}}$ as

$$
\begin{aligned}
& \psi_{1}=\sqrt{\left[\operatorname{Re}\left(\chi_{12}+\chi_{14}\right)\right]^{2}+\left[\operatorname{Im}\left(\chi_{12}+\chi_{14}\right)\right]^{2}} \\
& \psi_{2}=\sqrt{\left[\operatorname{Re}\left(\chi_{32}+\chi_{34}\right)\right]^{2}+\left[\operatorname{Im}\left(\chi_{32}+\chi_{34}\right)\right]^{2}} .
\end{aligned}
$$

where $\psi_{1}$ and $\psi_{2}$ represent the amplitudes of Py and Pmd layers, respectively. 

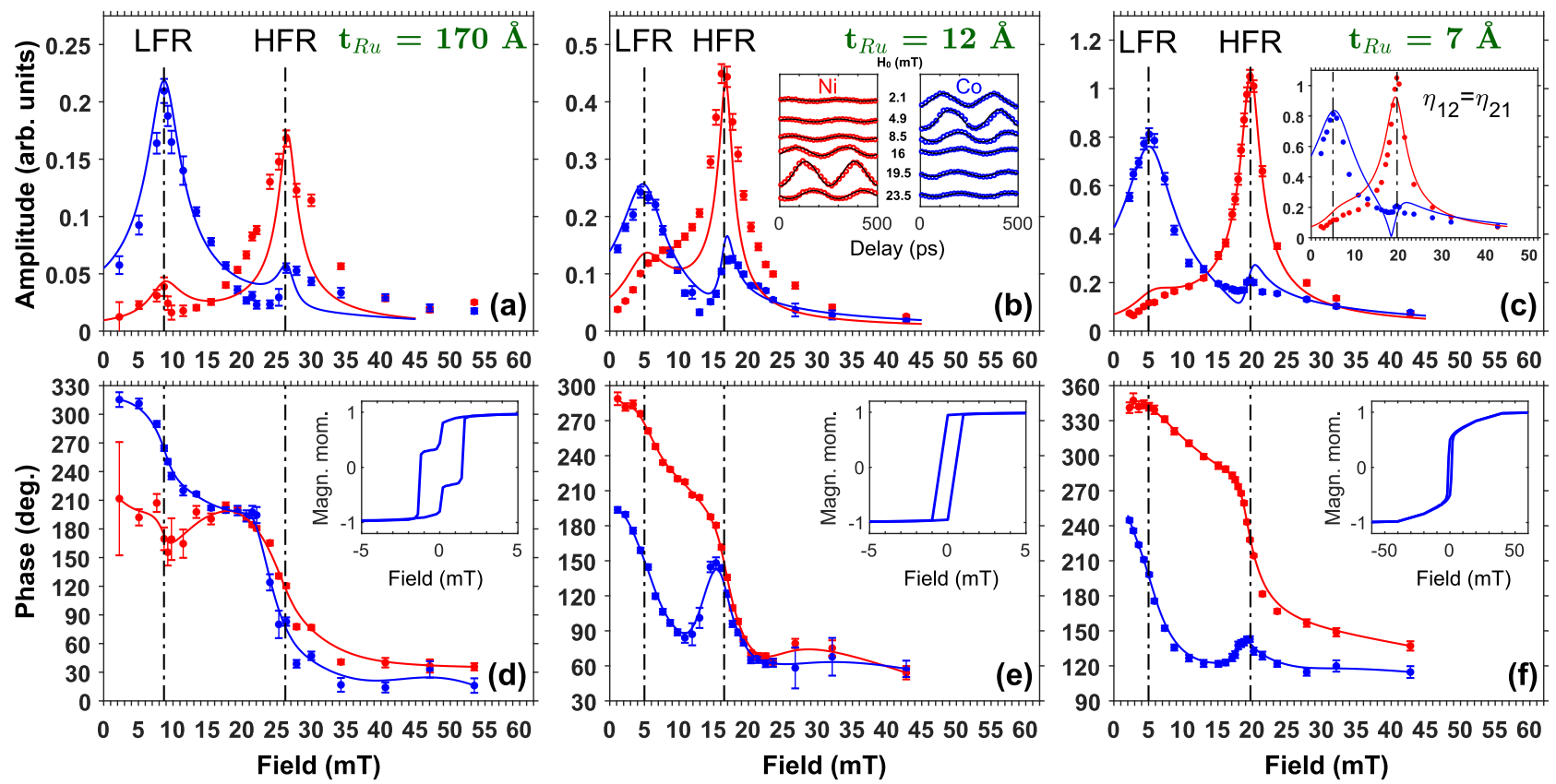

FIG. 2. Nonreciprocal spin-pumping damping theory and X-FMR measurements. Measured X-FMR amplitude and phase data (discrete points) acquired at an excitation frequency of $3.96 \mathrm{GHz}$ are shown as functions of applied field for three different Ru spacer layer thicknesses: (a) and (d) $170 \AA$, (b) and (e) $12 \AA$, and (c) and (f) $7 \AA$. The solid lines in the graphs showing the phase are high-order $B$ splines, whereas the solid lines for the amplitude response are model calculations based on Eq. (1). The Pmd layer response is shown in blue, whereas the Py is shown in red. The inset to (b) presents typical X-FMR delay scans for the Co and Ni edges acquired at different static bias fields. The inset in (c) shows model calculations for the $t_{\mathrm{Ru}} 7$ - $\AA$ sample with symmetric spin-pumping damping $\left(\eta_{12}=\eta_{21}=0.54\right)$. The insets in (d)-(f) present static magnetometry data, showing independent switching/no coupling [170 A (d)], ferromagnetic coupling [12 ̊ (e)], and biquadratic interaction $[7 \AA$ (f)].

\section{X-FMR MEASUREMENTS}

X-FMR [28-31] is the ideal technique to investigate nonreciprocal spin-pumping damping. Using X-FMR, it is feasible to measure the full complex susceptibility $\left(\chi^{\prime}\right.$ and $\left.\chi^{\prime \prime}\right)$ [32] or, equivalently, the amplitude and phase of oscillation resolved to individual elements and, hence, distinct magnetic layers [33-35]. X-FMR was used to determine the relative orientation of precessing spins in ferrimagnets [36] as well as to examine topics including spin pumping and the influence of spin currents [37-39]. For the investigation of nonreciprocal spin-pumping damping, the measured response of the individual layers can be compared directly with the equation of motion [Eq. (1)] for FM1 and FM2.

We use the X-FMR technique to study a series of $\mathrm{Py} / \mathrm{Ru} / \mathrm{Pmd}$ magnetic trilayer film structures. The film samples were fabricated at room temperature using dc magnetron sputtering (base pressure of $5 \times 10^{8}$ Torr) with the following structure: substrate/Ta(30 $\AA) / \operatorname{Py}(80 \AA) / \operatorname{Ru}\left(t_{\mathrm{Ru}}\right) /$ $\operatorname{Pmd}(80 \AA) / \mathrm{Ta}(30 \AA)$. Here, $t_{\mathrm{Ru}}$ varies between 7 and $170 \AA$. Single films of Pmd and Py with the same seed and cap layers were also fabricated for control measurements. The $\mathrm{Ru}$ spacer layer was deposited at low sputtering rate $(0.4 \AA / \mathrm{s})$ and low Ar gas pressure ( 3 mTorr) for optimal uniformity and interface smoothness. Composition and thickness of the films were verified using Rutherford backscattering spectrometry. Each sample was fabricated simultaneously on an oxidized $\mathrm{Si}$ substrate for magnetometry and structural measurements and on 100-nm-thick $\mathrm{Si}_{3} \mathrm{~N}_{4}$ membranes for X-FMR. To minimize the number of free parameters in Eq. (2), we conducted a series of static magnetometry and FMR measurements on the samples. In the X-FMR studies, the Ni in Py and Co in Pmd provide the elemental contrast to resolve the dynamics in the individual FM layers whereas Ru produces a strong interlayer exchange coupling that can be tuned from favoring parallel or antiparallel ground-state coupling as a function of the NM spacer thickness [40].

In X-FMR, we perform time-delay scans (equivalent to varying the phase between the sinusoidal RF excitation at $3.96 \mathrm{GHz}$ and the $\mathrm{x}$-ray bunches) with the photon energy tuned to the Ni or Co $L_{3}$ edge. The inset to Fig. 2(b) presents a subset of these delay scans; additional details on X-FMR are presented in Appendix C and in Ref. [32]. The amplitude and phase of the sinusoidal waveforms are extracted and plotted as discrete points in Figs. 2(a)-2(f). Also shown as insets to Figs. 2(d)-2(f) are normalized magnetization curves for the $t_{\mathrm{Ru}}=170,12$, and $7 \AA$ samples, showing the magnetic coupling is very weak [170 $\AA$, Fig. 2(d)], ferromagnetic [12 A, Fig. 2(e)], and biquadratic [7 А, Fig. 2(f)].

The $t_{\mathrm{Ru}}=170 \AA$ sample does not exhibit any bilinear or biquadratic interlayer exchange coupling, and the amplitude data in Fig. 2(a) reveal two resonances at $\sim 27 \mathrm{mT}$ and at $\sim 9 \mathrm{mT}$. The coupled nature of the dynamics of the FM layers precludes assignment of the combined resonance to a single layer, and we refer to these as a low-field resonance (LFR) and high-field resonance (HFR). The other samples also present a LFR and a HFR. At either resonant field, a particular FM layer does not respond independently; the other layer also 
exhibits a distinct, albeit weaker, response. This is clear, e.g., from Fig. 2(a) where the Py and Pmd layers have a maximum at the HFR and LFR, respectively. However, each layer has a second smaller peak in the amplitude that coincides with the resonance field of the other magnetic layer. This produces a nontrivial behavior of the amplitudes, originating from the coupling between the layers, and we observe a similar effect for all samples of this investigation.

The phase data also reveal a complex response of the oscillation phase as the magnetic field is swept through the resonance. For noninteracting layers, the phase of FM1 would change by $180^{\circ}$ when passing through the resonant field whereas the other layer would remain essentially unchanged. For coupled magnetic layers, the phases of the dynamic response would change according to Eq. (3). In the sample with the weakest coupling between Py and Pmd $\left[t_{\mathrm{Ru}}=170 \AA\right.$, Fig. 2(d)], the phase of the Py layer changes by $\sim 180^{\circ}$ through the HFR. However, the Pmd layer undergoes approximately the same phase shift through the HFR and then experiences an additional phase shift of $\sim 120^{\circ}$ through the LFR, indicating that there is sufficient coupling to cause a response distinctly different from that of noninteracting layers.

The variations in amplitude and phase are even more dramatic for the more strongly coupled samples $\left(t_{\mathrm{Ru}}=12\right.$ and $7 \AA$ ). In these systems, the phase of the Py layer shifts by about $180^{\circ}$ as the field is tuned from high fields down through the HFR; the Pmd phase initially follows the increase in phase, but once the HFR is passed, it falls off again. The effect is particularly pronounced in the FM-coupled $t_{\mathrm{Ru}}=$ $12 \AA$ sample where the two phases are essentially equal from the high field down through the HFR at $\sim 16 \mathrm{mT}$. As the field is reduced towards the LFR, the Pmd phase rises sharply to approach a value that is $\sim 120^{\circ}$ lower than that of the Py layer. For the $t_{\mathrm{Ru}}=7 \AA$ sample with biquadratic coupling, the phases of the two layers are distinctly different for all field strengths. This suggests, somewhat surprisingly, that the system with the closest distance between the Py and the Pmd layers, has the largest degree of independence in the magnetic response of both Py and Pmd.

\section{ANALYSIS AND DISCUSSION}

We begin our analysis with the phase data in Figs. 2(d)2(f). We fit the experimental phase data with a high-order $B$ spline [41] and use these interpolated values for the phase together with the parameters for magnetization, anisotropy, and interlayer exchange to calculate the amplitude response based on Eqs. (4) and (5). The results are presented as the solid lines in Figs. 2(a)-2(c). Apart from the phase data, all sample parameters used in the amplitude calculations (i.e., magnetization, anisotropy, layer thickness, exchange parameters, etc.) were obtained from independent magnetometry and conventional ferromagnetic resonance measurements (see Appendix D for details).

Generally, the theoretical model is in good agreement with the data in Fig. 2. The model captures the essential features of the Py and Pmd layers across both the LFR and the HFR; peak positions and amplitudes are well reproduced for the main resonance and the weaker response connected to the second layer. For example, in the coupled trilayer sample with the
TABLE I. Estimated spin-pumping-induced damping parameters and real part of the spin-mixing conductance in $\mathrm{cm}^{-2}$ for samples with $t_{\mathrm{Ru}}=7,12$, and $170 \AA$.

\begin{tabular}{lcccccc}
\hline \hline $\begin{array}{l}t_{\mathrm{Ru}} \\
\AA\end{array}$ & $\eta_{11}^{s p}$ & $\eta_{22}^{s p}$ & & & $\operatorname{Re}\left(g_{1}^{\uparrow \downarrow}\right)$ & $\begin{array}{c}\operatorname{Re}\left(g_{2}^{\uparrow \downarrow}\right) \\
10^{-4}\end{array}$ \\
\hline 7 & $10^{-3}$ & $\eta_{12}^{s p}$ & $\eta_{21}^{s p}$ & $10^{15}$ & 1.75 & 3.64 \\
12 & 3 & 1.6 & 0.47 & 0.61 & 11.75 \\
170 & 3 & 1.0 & 0.69 & 0.45 & 8.65 & 5.33 \\
\hline \hline
\end{tabular}

shortest interlayer distance [Fig. 2(c), $t_{\mathrm{Ru}}=7 \AA$ ], the model reproduces the increased amplitude in the response of the Pmd layer at the resonant field of the Py layer. For the ferromagnetically coupled sample [Fig. $2(\mathrm{~b}), t_{\mathrm{Ru}}=12 \AA$ ], the model accurately produces a reduced amplitude of the Pmd response at $5 \mathrm{mT}$ in comparison with the main Py resonance at $16 \mathrm{mT}$, in agreement with our experimental observations. The sample with the thickest $\mathrm{Ru}$ spacer [Fig. 2(a), $t_{\mathrm{Ru}}=170 \AA$ ] where interlayer exchange and dipolar coupling effects are negligible is particularly interesting. For completely decoupled layers, we would expect that the amplitude of the two resonances can be described by smooth symmetric Lorentzian functions. However, the experimental data show an increase in the Pmd (Py) amplitude at the resonant field of the Py (Pmd) layer and a deviation from Lorentzian shape, an effect that is clearly reproduced by the theory.

By using the measured phase response along with the independently derived material parameters, our model provides estimates of the precessional damping and the contributions from spin pumping (see Table I). $\operatorname{Re}\left(g_{i}^{\uparrow \downarrow}\right)$ (the real part of the spin-mixing conductance of layer $i$ ) is related to the dimensionless spin-pumping parameter $\eta_{i j}$ as [42]

$$
\operatorname{Re}\left(g_{i}^{\uparrow \downarrow}\right)=\frac{8 \pi m_{j}^{x} d_{j} \eta_{j i}}{g_{j} \mu_{B}}, \quad \text { where } i \neq j .
$$

Here, $g_{j}$ is the spectroscopic $g$ factor, and $\mu_{B}$ is the Bohr magneton. The contributions of the two interfaces (Py/Ru or $\mathrm{Ru} / \mathrm{Pmd}$ ) to the spin-mixing conductance are not reciprocal. For all the samples studied, we observe that the real part of the spin-mixing conductance from the Py layer that influences the Pmd layer is clearly bigger than the reversed spin-mixing conductance. The latter is close to values reported in the literature [42]. A symmetric spin-pumping damping model reproduces the data poorly as can be seen in the inset to Fig. 2(c), which assumes $\eta_{12}=\eta_{21}=0.54$. Apart from an overall poor description of the shape of the X-FMR data, such a model overestimates the magnitude of the Pmd (Py) response at most fields.

In the standard picture of spin pumping, a magnetic layer excited into precession drives a diffusive spin current in the direction transverse to the FM1/NM interface. The spin current incident upon the NM layer leads to spin accumulation in the NM near the interface and generates a flow of spin current back to the FM1. The spin-mixing conductance parametrizes the balance of the initial spin current $($ FM1 $\rightarrow$ NM) and the backflow into the magnetic layer. For NM layers that are thin compared to the spin-diffusion length in this layer $(\sim 150 \AA$ in $\mathrm{Ru}$ [43]), the spin current driven transports angular 
momentum across the NM/FM2 interface (with its own characteristic spin-mixing conductance) and thereby influencing the dynamics of FM2. The two spin-mixing conductances are often assumed to be equivalent [42]. However, the multilayer spin-pumping theory presented above together with the X-FMR data clearly indicate that spin-pumping damping is nonreciprocal (i.e., dependent on the direction of the spin current normal to the interface) in systems with nonequivalent interfaces [44-46]. The asymmetry of the spin-mixing conductance, albeit little discussed, is physically motivated, as it is proportional to the self-energy and the electronic structure of the respective magnetic layer [47]. There is no reason why this self-energy should be the same for Py and Pmd. In terms of symmetry, the nonreciprocity of the spin-pumping damping could be explained by breaking of the space inversion symmetry in the neighborhood of the interfaces induced by having two dissimilar ferromagnetic layers as was shown in Ref. [48] for the nonreciprocal magnon transport in synthetic magnets.

Interface spin transport governs a variety of phenomena, such as spin injection, the generation of pure spin currents, and the determination of spin Hall angles [3,49-51]. Spin pumping presents another method for manipulating magnetization across an interface, allowing for nonlocal effects. Spinpumping damping in magnetic trilayers has been examined previously in configurations with both parallel alignment and antiparallel moments canted in the direction of an external field $[52,53]$. Our analysis extends spin-pumping theory towards more general magnetic multilayer structures, which may have noncollinear or biquadratic interlayer exchange, different layer anisotropies, and distinctly different spin-mixing conductances. These effects will influence the dynamics of individual layers; however, when these issues are assessed independently, the asymmetry of the spin-pumping damping is revealed. Also, the description of magnetization dynamics in Eq. (1) is novel since it allows for spin pumping in noncollinear systems. In this paper, we report nonreciprocal spin-pumping damping in magnetic trilayers with dissimilar interfaces, and our findings may open possibilities in spintronics technology. Earlier first-principles calculations of spin pumping indicate that band matching across the interface (the matching of states in the NM with spin-resolved propagating states in the FM layer) greatly affects spin transmission and reflection across the interface [44,45]. Our analysis supports this viewpoint and shows that X-FMR can uniquely reveal differences in spin-pumping damping. Finally, we note that additional contributions to spin-pumping damping, such as spin-orbit coupling at the Ru/FM interfaces and spin current backflow variations for thick NM spacer layers, may affect the mutual spin dynamics in the FM layers. These contributions are being investigated with on-going first-principles calculations of the FM/NM interfaces, spin transport theory, and additional analytical modeling.

\section{ACKNOWLEDGMENTS}

The authors gratefully acknowledge support from the Knut and Alice Wallenberg Foundation, the Foundation for Strategic Research, eSSEENCE, StandUPP, the Swedish Energy Agency, the Swedish Research Council (VR) under Contracts No. 2016-04524 and No. 2013-08316. D.A.A. acknowledges

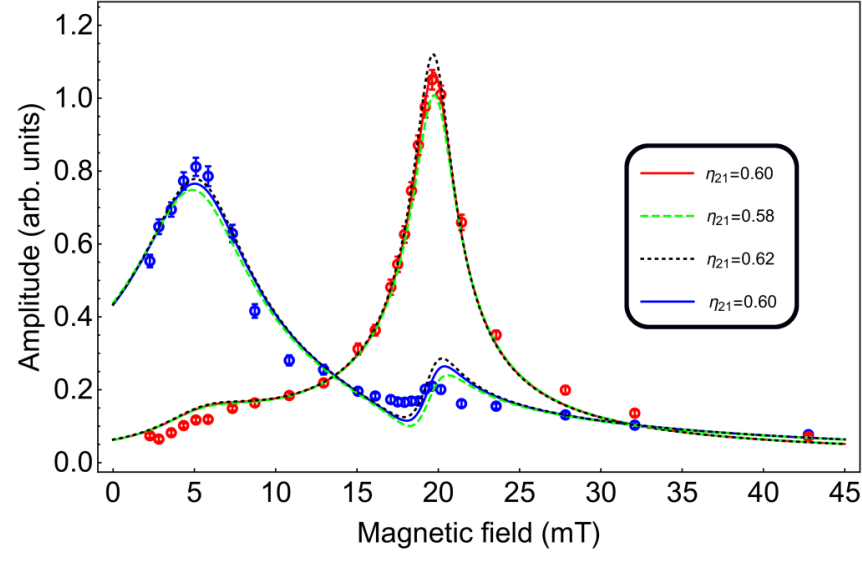

FIG. 3. Amplitude of the resonant response for the sample with Ru thickness of $7 \AA$. The spin-pumping damping $\eta_{21}$ has been varied whereas keeping constant the remaining parameters except for the dipolar field and the scaling parameter. The spin-pumping damping $\eta_{12}$ was fixed at 0.47 .

partial support through a University of South Florida Nexus Initiative (UNI) Award. This research used resources of the Advanced Photon Source, operated for the U.S Department of Energy Office of Science by Argonne National Laboratory under Contract No. DE-AC02-06CH11357.

\section{APPENDIX A: INFLUENCE OF SPIN-PUMPING DAMPING}

In Figs. 3 and 4, we show the influence of the variation of the spin-pumping damping on the amplitude of the resonant response for the particular case of the sample with Ru thickness of $7 \AA$. In general, a small variation of the spin-pumping damping produces a significant variation of the amplitude particularly around the resonance field. Since $\eta_{21}$ determines the spin pumping in the Pmd layer produced by the Py layer, the small resonant peak (or secondary peak) at around $20 \mathrm{mT}$ varies with $\eta_{21}$ [see Fig. 3, the dashed red and dotted black

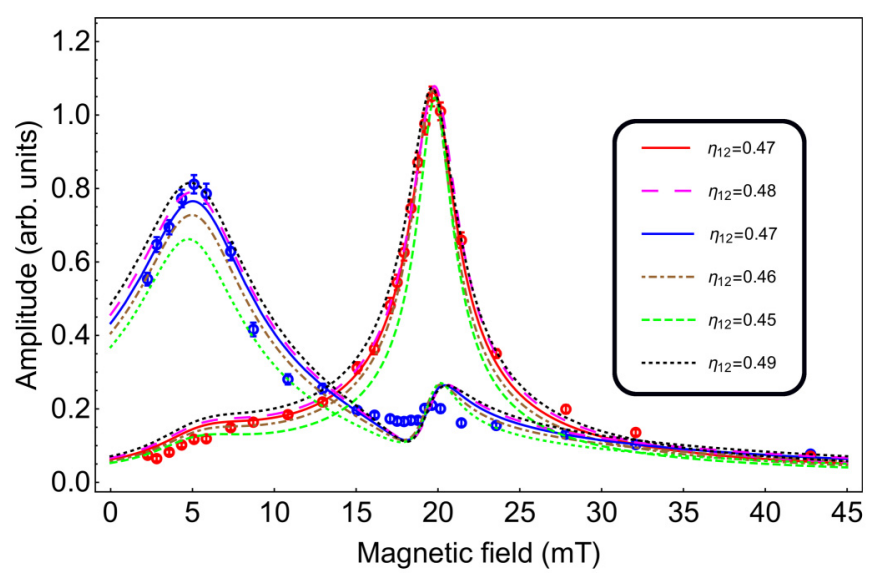

FIG. 4. Amplitude of the resonant response for the sample with Ru thickness of $7 \AA$. The spin-pumping damping $\eta_{12}$ has been varied whereas keeping constant the remaining parameters except for the dipolar field and the scaling parameter. The spin-pumping damping $\eta_{21}$ was fixed at 0.60 . 
lines], whereas the small resonant peak at around $5 \mathrm{mT}$ is unchanged. Note also that, if $\eta_{21}$ decreases, the secondary resonant peak also decreases and vice versa. The physical interpretation is as follows: By decreasing the spin-pumping damping, the Pmd layer experiences a smaller influence of the magnetization precession in the Py layer. The same line of reasoning is applied to the spin-pumping damping $\eta_{12}$ as shown in Fig. 4.

The FMR results are generally consistent with single-layer damping values presented in Table I. In particular, the FMR linewidth data indicate a higher damping value for the Pmd layer in the $t_{\mathrm{Ru}}=170-\AA$ sample as might be expected with a slightly rougher $\mathrm{Ru} / \mathrm{Pmd}$ interface with the thicker Ru spacer.

\section{APPENDIX B: NONRECIPROCAL SPIN-PUMPING DAMPING THEORY}

The magnetic susceptibility, which is a $4 \times 4$ matrix [according to Eq. (3) of the main part of the paper], is defined as

$$
\overline{\bar{\chi}}=\overline{\overline{\mathcal{A}}}^{-1},
$$

where the elements of the matrix $\overline{\overline{\mathcal{A}}}$ are as follows:

$$
\begin{aligned}
& a_{11}=\frac{-i \omega e^{i \phi_{1}}}{\gamma m_{1}^{x}} \\
& a_{12}=\left(-\frac{A_{e x}+2 B_{e x} \cos \beta}{\mu_{0} d_{1} m_{1}^{x 2}}-\frac{H_{0}-\delta_{1}}{m_{1}^{x}}-\frac{2 K_{11}^{c}}{\mu_{0} m_{1}^{x 2}}\right. \\
& \left.+\frac{2 K_{11}^{u}}{\mu_{0} m_{1}^{x 2}} \cos ^{2} \theta_{1}-\frac{\left(\eta_{1}^{0}+\eta_{11}^{s p}\right) i \omega}{\gamma m_{1}^{x}}\right) i e^{i \phi_{1}}, \\
& a_{13}=0 \text {, } \\
& a_{14}=\left(\frac{A_{e x}+2 B_{e x} \cos \beta}{\mu_{0} d_{1} m_{1}^{x} m_{2}^{x}}+\frac{\eta_{12}^{s p} i \omega}{\gamma m_{1}^{x}}\right) i e^{i \phi_{2}}, \\
& a_{21}=\left(\frac{2 K_{11}^{u}}{\mu_{0} m_{1}^{x 2}}\left(1-2 \cos ^{2} \theta_{1}\right)+\frac{2 K_{11}^{c}}{\mu_{0} m_{1}^{x 2}}+\frac{A_{e x}+2 B_{e x} \cos \beta}{\mu_{0} d_{1} m_{1}^{x 2}}\right. \\
& \left.+\frac{H_{0}-\delta_{1}}{m_{1}^{x}}+\frac{\left(\eta_{1}^{0}+\eta_{11}^{s p}\right) i \omega}{\gamma m_{1}^{x}}\right) e^{i \phi_{1}}, \\
& a_{22}=\frac{\omega e^{i \phi_{1}}}{\gamma m_{1}^{x}}, \\
& a_{23}=\left(-\frac{A_{e x}+2 B_{e x} \cos \beta}{\mu_{0} d_{1} m_{1}^{x} m_{2}^{x}}-\frac{\eta_{12}^{s p} i \omega}{\gamma m_{1}^{x}}\right) e^{i \phi_{2}}, \\
& a_{24}=0 \text {, } \\
& a_{31}=0 \text {, } \\
& a_{32}=\left(\frac{A_{e x}+2 B_{e x} \cos \beta}{\mu_{0} d_{2} m_{1}^{x} m_{2}^{x}}+\frac{\eta_{21}^{s p} i \omega}{\gamma m_{2}^{x}}\right) i e^{i \phi_{1}}, \\
& a_{33}=\frac{-i \omega e^{i \phi_{2}}}{\gamma m_{2}^{x}} \text {, } \\
& a_{34}=\left(\frac{-2 K_{12}^{c}}{\mu_{0} m_{2}^{x 2}}-\frac{A_{e x}+2 B_{e x} \cos \beta}{\mu_{0} d_{2} m_{2}^{x 2}}-\frac{H_{0}-\delta_{2}}{m_{2}^{x}}\right. \\
& \left.+\frac{2 K_{12}^{u} \cos ^{2} \theta_{2}}{\mu_{0} m_{2}^{x 2}}-\frac{\left(\eta_{2}^{0}+\eta_{22}^{s p}\right) i \omega}{\gamma m_{2}^{x}}\right) i e^{i \phi_{2}},
\end{aligned}
$$

\begin{tabular}{|c|c|c|c|c|}
\hline$t_{\mathrm{Ru}}$ & $\begin{array}{c}A_{e x} \\
\left(\mathrm{~J} / \mathrm{m}^{2}\right)\end{array}$ & $\begin{array}{c}B_{e x} \\
\left(\mathrm{~J} / \mathrm{m}^{2}\right)\end{array}$ & $\eta_{1}$ & $\eta_{2}$ \\
\hline 7 & 0 & $-10.5 \times 10^{-5}$ & 0.00852 & 0.00426 \\
\hline 12 & $4.5 \times 10^{-5}$ & 0 & 0.00852 & 0.00442 \\
\hline 170 & 0 & 0 & 0.00852 & 0.00640 \\
\hline
\end{tabular}

TABLE II. Estimated exchange constants and intrinsic dampings for samples with $t_{\mathrm{Ru}}=7,12$, and $170 \AA$.

$$
\begin{aligned}
a_{41}= & \left(-\frac{A_{e x}+2 B_{e x} \cos \beta}{\mu_{0} d_{2} m_{1}^{x} m_{2}^{x}}-\frac{\eta_{11}^{s p} i \omega}{\gamma m_{2}^{x}}\right) e^{i \phi_{1}}, \\
a_{42}= & 0, \\
a_{43}= & \left(\frac{2 K_{12}^{u}}{\mu_{0} m_{2}^{x 2}}\left(1-2 \cos ^{2} \theta_{2}\right)+\frac{2 K_{12}^{c}}{\mu_{0} m_{2}^{x 2}}+\frac{A_{e x}+2 B_{e x} \cos \beta}{\mu_{0} d_{2} m_{2}^{x 2}}\right. \\
& \left.+\frac{H_{0}-\delta_{2}}{m_{2}^{x}}-\frac{\left(\eta_{2}^{0}+\eta_{22}^{s p}\right) i \omega}{\gamma m_{2}^{x}}\right) e^{i \phi_{2}}, \\
a_{44}= & \frac{\omega e^{i \phi_{2}}}{\gamma m_{2}^{x}} .
\end{aligned}
$$

The angle between the direction of the magnetization in the Py layer with respect to the magnetization in the Pmd layer is indicated here by the symbol $\beta$. By using the data collected in Tables I and II and the measured phase shown in Fig. 2, the model described by Eq. (3) provides the spin-pumping dampings, angle $\beta$, and dipolar field prefactors $\left(\delta_{i}\right)$. These data are collected in Tables I and III.

\begin{tabular}{|c|c|c|c|}
\hline$t_{\mathrm{Ru}}$ & $\begin{array}{c}\beta \\
\mathrm{rad}\end{array}$ & $\begin{array}{c}\delta_{1} \\
\mathrm{mT}\end{array}$ & $\begin{array}{c}\delta_{2} \\
\mathrm{mT}\end{array}$ \\
\hline 7 & 2 & -117.78 & 111.88 \\
\hline 12 & 0 & -91.83 & 121.56 \\
\hline 170 & 0 & -124.02 & 156.29 \\
\hline
\end{tabular}

\section{APPENDIX C: X-FMR MEASUREMENTS}

X-ray detected ferromagnetic resonance [28-31], or XFMR, is the ideal technique to investigate nonreciprocal spinpumping damping. X-FMR combines $\mathrm{X}$-ray magnetic circular dichroism (XMCD) with FMR, and an overview of the technique is presented in Fig. 5. As with measurements of element-specific hysteresis loops, tuning the x-ray energy to the absorption edge of different elements (e.g., the Co or $\mathrm{Ni}$ $L_{3}$ edges) isolates the magnetic contribution from a single layer in a multilayer magnetic structure. And by using a microwave FMR excitation that is phase locked with the x-ray bunch clock at a synchrotron storage ring, the full complex susceptibility ( $\chi^{\prime}$ and $\left.\chi^{\prime \prime}\right)$ [32] or, equivalently, the amplitude and the phase of the FMR oscillation is resolved to individual elements and, hence, distinct magnetic layers [33-35]. XFMR was previously used to determine the relative orientation

TABLE III. Angle between the direction of the magnetization in the Py layer with respect to the magnetization in the Pmd layer as well as the layer-dependent dipolar field prefactor for samples with $t_{\mathrm{Ru}}=7,12$, and $170 \AA$. 


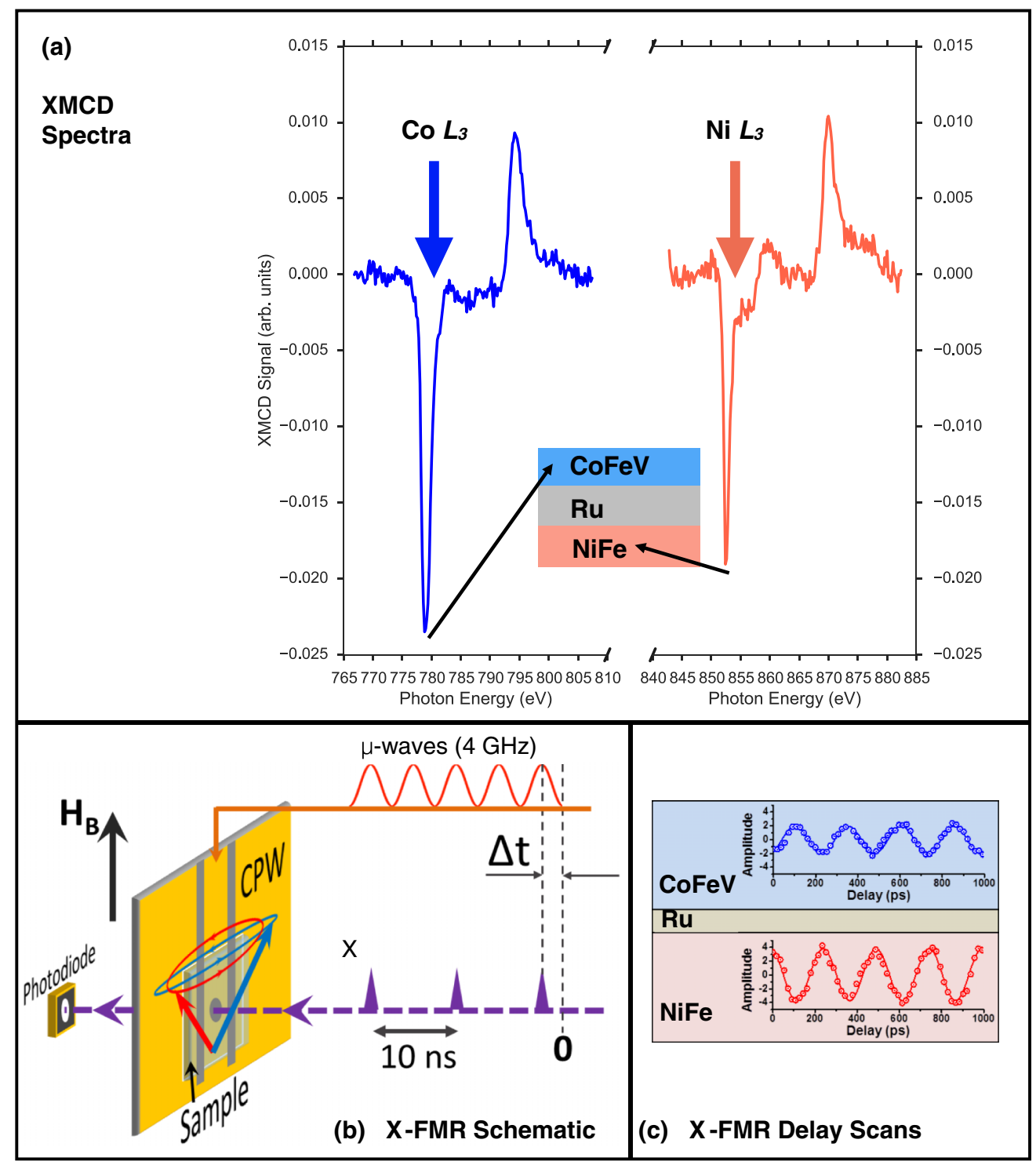

FIG. 5. X-FMR principle and setup. (a) XMCD spectra from a Pmd/Ru/Py trilayer. By tuning the photon energy to the Co (Ni) $L_{3}$ edge, the magnetization dynamics of the Pmd (Py) layer can be isolated from the collective response. (b) X-FMR scans are conducted in X-ray transmission mode. The sample is placed on a co-planar waveguide, and the microwave excitation is synchronized with the $\mathrm{x}$-ray bunches. A digital delay generator (not shown) varies the phase between the x-ray bunches and the rf excitation. (c) Typical delay scans from the Pmd or Py layer are sinusoidal functions from which the amplitude and relative phase can be extracted.

of precessing spins in ferrimagnets [36] as well as to examine topics including spin pumping and the influence of spin currents $[37,38,54,55]$. For the investigation of nonreciprocal spin-pumping damping, the measured motion of the individual layers via X-FMR can then be compared directly with results that come from the equation of motion [Eq. (1)] for FM1 and FM2.

In our implementation of the technique, X-FMR is a pumpprobe measurement where the pump is a sinusoidal rf signal that is phase locked to the photon bunch repetition frequency of the synchrotron. The probe is the circularly polarized $\mathrm{x}$ rays whose energy is tuned to the $L_{3}$ absorption edge of Co for sensitivity to the Pmd layer or Ni for the Py layer [see Fig. 5(a)]. The thin-film sample is placed on a custom co-planar waveguide that permits transmission of the incident $\mathrm{x}$ rays. The $\mathrm{rf}$ signal excites precession of the magnetization in the magnetic layers, and the $\mathrm{x}$ rays transmitted through the sample are detected with a photodiode [Fig. 5(b)]. We conducted the X-FMR measurements at beamline 4-ID-C of the Advanced Photon Source (Argonne National Laboratory, Argonne, IL). In X-FMR experiments, we perform time-delay scans, which are equivalent to varying the phase between the sinusoidal rf signal and the arrival of the x-ray photons. Figure 5(c) presents a subset of these delay scans; for further details, refer to Ref. [29]. The x-ray photon bunches with a bunch length of $\sim 60 \mathrm{ps}$ [32] sample the projection of the magnetization along the beam propagation direction as a function of time delay or phase. The simple sinusoidal waveforms of the delay scans allow us to extract the amplitude of the precessional motion and phase relative to the microwave field of the pump. We use a fixed frequency of $3.96 \mathrm{GHz}$ (the 45th harmonic of the $88-\mathrm{MHz}$ bunch repetition frequency 
of the synchrotron) and recorded the response of the Pmd and Py layers as we varied the static bias field $H_{B}$ through both the high- and the low-field resonances. The resulting amplitude and phase data for the three samples are shown in Figs. 2(a)-2(f) of the main text as discrete points.

\section{APPENDIX D: STATIC MAGNETOMETRY AND FERROMAGNETIC RESONANCE MEASUREMENTS}

Field hysteresis measurements of single magnetic layers of the control samples provided the saturation magnetization for each magnetic layer: $4 \pi m_{\mathrm{Py}}=0.89 \times 10^{7}$ and $4 \pi m_{\mathrm{Pmd}}=$ $2.25 \times 10^{7} \mathrm{~A} / \mathrm{m}$. Magnetization curves for a selected number of samples with $t_{\mathrm{Ru}}=7,12,170 \AA$ are shown as insets in Figs. 2(f), 2(e) and 2(d), respectively. The $t_{\mathrm{Ru}}=7-\AA$ sample shows the behavior typical for a $90^{\circ}$ coupling between Py and Pmd layers. A Ru thickness of $t_{\mathrm{Ru}}=12 \AA$ favors FM coupling between magnetic layers, and for a thick Ru spacer $\left(t_{\mathrm{Ru}}=\right.$ $170 \AA$ A) the magnetic layers are decoupled. Magnetometry results correlate well with the FMR measurements.

We measured the in-plane uniaxial anisotropy constants with angular-dependent $X$-band $(9.8-\mathrm{GHz})$ FMR (rotation about the surface normal) whereas the interlayer exchange constants $\left(A_{e x}\right.$ and $\left.B_{e x}\right)$ were determined from in-plane FMR measurements at varying excitation frequencies $(2-12 \mathrm{GHz})$. The uniaxial anisotropy field is in-plane, i.e., it lies on the $x-y$ plane. Here, $\theta_{i}$ is the angle between the uniaxial easy axis and the magnetization at layer $i$. Then, the dependence of the uniaxial anisotropy field on the $\theta_{i}$ angle is $\left(\boldsymbol{m}_{i} \cdot \widehat{\boldsymbol{e}}_{u}\right) \widehat{\boldsymbol{e}}_{u}=$ $\left(m_{i}^{x} \cos \theta_{i}+m_{i}^{y} \sin \theta_{i}\right)\left(\cos \theta_{i}, \sin \theta_{i}, 0\right)$. In the samples considered here, the uniaxial anisotropy is along the $x$ axis, i.e., $\theta_{i}=0$. The angular-dependent $X$-band measurements indicate that all samples exhibit a weak uniaxial anisotropy with the largest anisotropy constant at about $K_{\mathrm{Pmd}}^{u}=2408 \mathrm{~J} / \mathrm{m}^{3}$
TABLE IV. Measured physical magnitudes for Py and Pmd layers.

\begin{tabular}{lccccc}
\hline \hline & $K_{i}^{u}$ & $K_{i}^{c}$ & $d_{i}$ & $m_{i}^{x}$ & $\omega$ \\
Layer & $\left(\mathrm{J} / \mathrm{m}^{3}\right)$ & $\left(\mathrm{J} / \mathrm{m}^{3}\right)$ & $(\mathrm{m})$ & $(\mathrm{A} / \mathrm{m})$ & $(\mathrm{GHz})$
\end{tabular}

Py $(i=1) \quad 184.14210 .6248 \times 10^{-9}\left(0.89 \times 10^{7}\right) /(4 \pi) 3.96$ Pmd $(i=2) 2408.213179 .0498 \times 10^{-9}\left(2.25 \times 10^{7}\right) /(4 \pi) 3.96$

for Pmd and $K_{\mathrm{Py}}^{u}=184 \mathrm{~J} / \mathrm{m}^{3}$ for Py. Cubic anisotropy was found to be negligibly small: $K_{\mathrm{Pmd}}^{c}=179 \mathrm{~J} / \mathrm{m}^{3}$ and $K_{\mathrm{Py}}^{c}=$ $10.6 \mathrm{~J} / \mathrm{m}^{3}$. Note that we consider the anisotropy constants as independent of the spacer layer thickness. All measured parameters are summarized in Table IV.

We determined $A_{e x}$ and $B_{e x}$ from fits of the resonant field vs frequency as outlined in Ref. [56]. The sample with the thickest NM spacer layer $\left(t_{\mathrm{Ru}}=170 \AA\right)$ does not present any bilinear or biquadratic coupling, consistent with the $M$ vs $H$ loops which show the switching of the individual layers [see the inset in Fig. 2(d)]. As the Ru thickness decreases, interlayer-exchange coupling begins to correlate the switching of the two layers. The $t_{\mathrm{Ru}}=12-\AA$ sample shows FM coupling between magnetic layers with only a bilinear type of coupling present $A_{e x}=4.5 \times 10^{-5} \mathrm{~J} / \mathrm{m}^{2}$; the field hysteresis loops confirm this as only a single switching field is evident [see the inset in Fig. 2(e)]. Finally, for the $t_{\mathrm{Ru}}=7 \AA$ sample we find the bilinear exchange coupling constant $A_{e x}=0$, although there is a large biquadratic coupling parameter $B_{e x}=$ $-10.5 \times 10^{-5} \mathrm{~J} / \mathrm{m}^{2}$. This indicates that the coupling of the two layers is shifting from FM to AFM, leaving a $\sim 90^{\circ}$ coupling between the Py and the Pmd layers. Exchange constants together with the intrinsic dampings are summarized in Table II.
[1] K. Uchida, S. Takahashi, K. Harii, J. Ieda, W. Koshibae, K. Ando, S. Maekawa, and E. Saitoh, Nature (London) 455, 778 (2008).

[2] J. E. Hirsch, Phys. Rev. Lett. 83, 1834 (1999).

[3] C.-F. Pai, L. Liu, Y. Li, H. W. Tseng, D. C. Ralph, and R. A. Buhrman, Appl. Phys. Lett. 101, 122404 (2012).

[4] N. Okuma, M. R. Masir, and A. H. MacDonald, Phys. Rev. B 95, 165418 (2017).

[5] L. J. Cornelissen, J. Liu, R. A. Duine, J. B. Youssef, and B. J. van Wees, Nat. Phys. 11, 1022 (2015).

[6] B. L. Giles, Z. Yang, J. S. Jamison, and R. C. Myers, Phys. Rev. B 92, 224415 (2015).

[7] W. Yuan, Q. Zhu, T. Su, Y. Yao, W. Xing, Y. Chen, Y. Ma, X. Lin, J. Shi, R. Shindou, X. C. Xie, and W. Han, Sci. Adv. 4, eaat1098 (2018).

[8] J. C. Slonczewski, J. Magn. Magn. Mater. 159, L1 (1996).

[9] L. Berger, Phys. Rev. B 54, 9353 (1996).

[10] T. Chen, R. K. Dumas, A. Eklund, P. K. Muduli, A. Houshang, A. A. Awad, P. Durrenfeld, B. G. Malm, A. Rusu, and J. Åkerman, Proc. IEEE 104, 1919 (2016).

[11] F. Hellman, A. Hoffmann, Y. Tserkovnyak, G. S. D. Beach, E. E. Fullerton, C. Leighton, A. H. MacDonald, D. C. Ralph, D. A. Arena, H. A. Dürr, P. Fischer, J. Grollier, J. P. Heremans,
T. Jungwirth, A. V. Kimel, B. Koopmans, I. N. Krivorotov, S. J. May, A. K. Petford-Long, J. M. Rondinelli, N. Samarth, I. K. Schuller, A. N. Slavin, M. D. Stiles, O. Tchernyshyov, A. Thiaville, and B. L. Zink, Rev. Mod. Phys. 89, 025006 (2017).

[12] Y. Tserkovnyak, A. Brataas, and G. E. W. Bauer, Phys. Rev. B 66, 224403 (2002).

[13] M. R. Sears and W. M. Saslow, Phys. Rev. B 85, 014404 (2012).

[14] E. Saitoh, M. Ueda, H. Miyajima, and G. Tatara, Appl. Phys. Lett. 88, 182509 (2006).

[15] S. Mizukami, Y. Ando, and T. Miyazaki, J. Magn. Magn. Mater. 239, 42 (2002).

[16] B. Heinrich, G. Woltersdorf, R. Urban, and E. Simanek, J. Appl. Phys. 93, 7545 (2003).

[17] Y. Tserkovnyak, A. Brataas, and G. E. W. Bauer, Phys. Rev. Lett. 88, 117601 (2002).

[18] B. Heinrich, Y. Tserkovnyak, G. Woltersdorf, A. Brataas, R. Urban, and G. E. W. Bauer, Phys. Rev. Lett. 90, 187601 (2003).

[19] H. Yang, Y. Li, and W. E. Bailey, Appl. Phys. Lett. 108, 242404 (2016).

[20] M. Wang, W. Cai, K. Cao, J. Zhou, J. Wrona, S. Peng, H. Yang, J. Wei, W. Kang, Y. Zhang, J. Langer, B. Ocker, A. Fert, and W. Zhao, Nat. Commun. 9, 671 (2018). 
[21] S. Okamoto, N. Kikuchi, M. Furuta, O. Kitakami, and T. Shimatsu, J. Phys. D: Appl. Phys. 48, 353001 (2015).

[22] A. M. Deac, A. Fukushima, H. Kubota, H. Maehara, Y. Suzuki, S. Yuasa, Y. Nagamine, K. Tsunekawa, D. D. Djayaprawira, and N. Watanabe, Nat. Phys. 4, 803 (2008).

[23] S. M. Mohseni, S. R. Sani, J. Persson, T. N. A. Nguyen, S. Chung, Y. Pogoryelov, P. K. Muduli, E. Iacocca, A. Eklund, R. K. Dumas, S. Bonetti, A. Deac, M. A. Hoefer, and J. Akerman, Science 339, 1295 (2013).

[24] S. Sani, J. Persson, S. Mohseni, Y. Pogoryelov, P. Muduli, A. Eklund, G. Malm, M. Käll, A. Dmitriev, and J. Åkerman, Nat. Commun. 4, 2731 (2013).

[25] I. McFadyen, E. Fullerton, and M. Carey, MRS Bull. 31, 379 (2006).

[26] E. Tsymbal, J. Magn. Magn. Mater. 130, L6 (1994).

[27] Note that, in Eqs. (4) and (5), the subindices of the susceptibility refer to its matrix elements.

[28] W. E. Bailey, L. Cheng, D. J. Keavney, C.-C. Kao, E. Vescovo, and D. A. Arena, Phys. Rev. B 70, 172403 (2004).

[29] T. Martin, G. Woltersdorf, C. Stamm, H. A. Durr, R. Mattheis, C. H. Back, and G. Bayreuther, J. Appl. Phys. 105, 07D310 (2009).

[30] M. K. Marcham, P. S. Keatley, A. Neudert, R. J. Hicken, S. A. Cavill, L. R. Shelford, G. van der Laan, N. D. Telling, J. R. Childress, J. A. Katine, P. Shafer, and E. Arenholz, J. Appl. Phys. 109, 07D353 (2011).

[31] P. Warnicke, R. Knut, E. Wahlström, O. Karis, W. E. Bailey, and D. A. Arena, J. Appl. Phys. 113, 033904 (2013).

[32] D. A. Arena, Y. Ding, E. Vescovo, S. Zohar, Y. Guan, and W. E. Bailey, Rev. Sci. Instrum. 80, 083903 (2009).

[33] D. A. Arena, E. Vescovo, C.-C. Kao, Y. Guan, and W. E. Bailey, Phys. Rev. B 74, 064409 (2006).

[34] Y. Guan, W. E. Bailey, C.-C. Kao, E. Vescovo, and D. A. Arena, J. Appl. Phys. 99, 08J305 (2006).

[35] W. E. Bailey, C. Cheng, R. Knut, O. Karis, S. Auffret, S. Zohar, D. Keavney, P. Warnicke, J.-S. Lee, and D. A. Arena, Nat. Commun. 4, 2025 (2013).

[36] P. Warnicke, E. Stavitski, J.-S. Lee, A. Yang, Z. Chen, X. Zuo, S. Zohar, W. E. Bailey, V. G. Harris, and D. A. Arena, Phys. Rev. B 92, 104402 (2015).

[37] J. Li, L. R. Shelford, P. Shafer, A. Tan, J. X. Deng, P. S. Keatley, C. Hwang, E. Arenholz, G. van der Laan, R. J. Hicken, and Z. Q. Qiu, Phys. Rev. Lett. 117, 076602 (2016).
[38] A. A. Baker, A. I. Figueroa, L. J. Collins-McIntyre, G. van der Laan, and T. Hesjedal, Sci. Rep. 5, 7907 (2015).

[39] A. Figueroa, A. Baker, L. Collins-McIntyre, T. Hesjedal, and G. van der Laan, J. Magn. Magn. Mater. 400, 178 (2016).

[40] S. S. P. Parkin, Phys. Rev. Lett. 67, 3598 (1991).

[41] C. de Boor, A Practical Guide to Splines (Springer-Verlag, New York, 1978).

[42] C. J. Durrant, L. R. Shelford, R. A. J. Valkass, R. J. Hicken, A. I. Figueroa, A. A. Baker, G. van der Laan, L. B. Duffy, P. Shafer, C. Klewe, E. Arenholz, S. A. Cavill, J. R. Childress, and J. A. Katine, Phys. Rev. B 96, 144421 (2017).

[43] N. Behera, M. S. Singh, S. Chaudhary, D. K. Pandya, and P. K. Muduli, J. Appl. Phys. 117, 17A714 (2015).

[44] M. Zwierzycki, Y. Tserkovnyak, P. J. Kelly, A. Brataas, and G. E. W. Bauer, Phys. Rev. B 71, 064420 (2005).

[45] K. Xia, P. J. Kelly, G. E. W. Bauer, A. Brataas, and I. Turek, Phys. Rev. B 65, 220401(R) (2002).

[46] Y. Liu, Z. Yuan, R. J. H. Wesselink, A. A. Starikov, and P. J. Kelly, Phys. Rev. Lett. 113, 207202 (2014).

[47] K. Carva and I. Turek, Phys. Rev. B 76, 104409 (2007).

[48] J. Chen, T. Yu, C. Liu, T. Liu, M. Madami, K. Shen, J. Zhang, S. Tu, M. S. Alam, K. Xia, M. Wu, G. Gubbiotti, Y. M. Blanter, G. E. W. Bauer, and H. Yu, Phys. Rev. B 100, 104427 (2019).

[49] P. Dürrenfeld, F. Gerhard, J. Chico, R. K. Dumas, M. Ranjbar, A. Bergman, L. Bergqvist, A. Delin, C. Gould, L. W. Molenkamp, and J. Åkerman, Phys. Rev. B 92, 214424 (2015).

[50] N. Roschewsky, T. Matsumura, S. Cheema, F. Hellman, T. Kato, S. Iwata, and S. Salahuddin, Appl. Phys. Lett. 109, 112403 (2016).

[51] W. Zhang, W. Han, X. Jiang, S.-H. Yang, and S. S. P. Parkin, Nat. Phys. 11, 496 (2015).

[52] S. Takahashi, Appl. Phys. Lett. 104, 052407 (2014).

[53] T. Chiba, G. E. W. Bauer, and S. Takahashi, Phys. Rev. B 92, 054407 (2015).

[54] A. A. Baker, A. I. Figueroa, C. J. Love, S. A. Cavill, T. Hesjedal, and G. van der Laan, Phys. Rev. Lett. 116, 047201 (2016).

[55] A. A. Baker, A. I. Figueroa, D. Pingstone, V. K. Lazarov, G. Van Der Laan, and T. Hesjedal, Sci. Rep. 6, 35582 (2016).

[56] Y. Wei, S. Jana, R. Brucas, Y. Pogoryelov, M. Ranjbar, R. K. Dumas, P. Warnicke, J. Akerman, D. A. Arena, O. Karis, and P. Svedlindh, J. Appl. Phys. 115, 17D129 (2014). 